\title{
Added Mass of High-Altitude Balloons
}

\author{
W. J. Anderson, ${ }^{*}$ G. N. Shah, $\nmid$ and Jungsun Park \\ University of Michigan, Ann Arbor, Michigan 48109
}

\begin{abstract}
Acoustic theory is used to find the added mass for several rigid, immersed bodies. The classical cases of a thin circular disk and a sphere are used to determine the mesh fineness required for engineering accuracy. A family of five Smalley-shaped balloons (zero circumferential stress in the film) is then considered, at different inflation ratios. Acoustical boundary elements are used. The fluid is assumed incompressible and, therefore, the added masses are identical in spirit with those from hydrodynamics. Although not important for this study, compressibility effects can be included for other bodies, if needed. Both vertical and horizontal accelerations are considered. Results show that the pear-shaped balloons behave in an intermediate way between spheres and cylinders, as expected. Such accurate values for added mass will allow better simulation of balloon flight, particularly for dynamic motion resulting from ballast or payload drop. A major feature of this article is to demonstrate the feasibility of calculating added masses for arbitrarily shaped bodies using acoustics. The authors feel this approach will become a standard working tool for studies of immersed bodies such as balloons, parachutes, and submarines because of the ease of computation. The method uses commercial finite element preprocessors for building the model, calculation of enclosed volumes, and transferring rigid body information to the acoustic computer program.
\end{abstract}

\section{Nomenclature}

$[C]=$ coupling matrix

$F_{1}=$ acoustic load vector

$\boldsymbol{F}_{\mathrm{S}}=$ structural force vector

$[H]=$ fluid matrix

$[K]=$ stiffness matrix

$k=$ wave number

$[M]=$ mass matrix

$p=$ acoustic pressure

$\bar{p}=$ prescribed acoustic pressure on surface $S_{1}$

$\{Q\}=$ potential (jump of pressure) vector

$r^{2}=$ coefficient of determination of curve fit

$S_{1} \quad=$ surface where pressures $\bar{p}$ are specified

$S_{2} \quad=$ surface where velocities $\bar{v}_{n}$ are specified

$\{\overline{\boldsymbol{U}}\}=$ displacement vector

$v \quad=$ normal velocity

$\bar{v}=$ prescribed normal velocity on surface $S_{2}$

$y(x)=$ polynomial curve fit for inertia coefficient

$\rho=$ fluid density

$\omega=$ frequency, $\mathrm{rad} / \mathrm{s}$

\section{Subscripts}

$A \quad=$ added mass

$F \quad=$ fluid

$n=$ normal

$S \quad=$ structural

$$
\begin{aligned}
& \text { Superscripts } \\
& \begin{aligned}
T \quad= & \text { transpose } \\
-1 & =\text { inverse } \\
-\quad & =\text { given value of variable, e.g., pressure }
\end{aligned}
\end{aligned}
$$

Prescnted as Paper 91-3693 at the AIAA International Balloon Technology Conference, Albuquerque, Oct. 8-10, 1991; received May 5, 1992; revision received Dec. 17, 1993; accepted for publication May 28, 1994. Copyright (C) 1994 by the authors. Published by the American Institute of Aeronautics and Astronautics, Inc., with permission.

"Professor of Aerospace Engineering. Senior Member AIAA.

†raduate Student, Aerospace Engineering. Student Member AIAA.

$\ddagger$ Graduate Student, Acrospace Engineering. Member AIAA.

\section{Introduction}

$\mathrm{F}$ a solid body is immersed in a fluid and is then accelerated, pressures are generated that affect the fluid field to infinity. This creates kinetic energy in the fluid. One can define an effective mass of fluid accelerating with the body; this is called "added" mass. Historically, the calculation of added mass has been in the province of hydrodynamics.

Added masses are very important in studying dynamics of bodies that are submerged in relatively heavy fluids. Neutrally buoyant bodies such as balloons and submarines certainly qualify, as well as bodies that have large paddle-shaped surfaces that disturb the fluid, such as a thin disk oscillating normal to its surface.

Much recent progress in the area of numerical modeling of coupled acoustics/structures has been made. ${ }^{1.3}$ Major software packages use boundary elements for the fluid discretization. ${ }^{+}$This modeling procedure can be used to determine the added mass due to rigid-body structural accelerations. The effect of the pressure field is to retard the acceleration of the body, and the resulting added mass of the body can then be deduced. This capability has not existed before, and promises to aid the study of bodies such as balloons, parachutes, and submarines where the bodies carry a large amount of fluid with them.

\section{Eigenvalue Equations}

This study is based on a solution of the Helmholtz equation. One can solve this acoustic equation either by a potential field solution or by a pressure field solution. The present approach is to consider the pressure field as the primary variable and to cast boundary conditions into functions of pressure. The Helmholtz equation is

$$
\nabla^{2} p+k^{2} p=0
$$

and the associated boundary conditions are

$$
\begin{aligned}
p=\bar{p}(x, y, z) & \text { (on surface } \left.S_{1}\right) \\
\boldsymbol{v}_{n}=\bar{v}_{n}(x, y, z) & \text { (on surface } \left.S_{2}\right)
\end{aligned}
$$

In addition, one has the Sommerfeld radiation condition for 
unbounded domains:

$$
\lim _{x \rightarrow \infty} r^{2}\left(\frac{\partial p}{\partial n}+i k p\right)=0
$$

The solution to these equations will be carried out for various-shaped bodies using a commercial computer program, SYSNOISE. ${ }^{4}$ Quadrilateral and triangular elements are used. It is a peculiarity of the boundary elements used that the fluid pressure exists on both sides of the element. For hollow bodies, such as the balloon, the elements therefore model the (same) fluid on both the interior and exterior of the body. For the balloon, the interior air is spurious and its contribution to the added mass must be removed and replaced by the mass of helium actually present. This correction requires accurate knowledge of the enclosed volume of the balloon and this is provided by the preprocessor.

After the fluid field is modeled by boundary elements, the structure is modeled by conventional finite elements. The translational rigid body modes of the structure are easily represented by commercial finite element programs, which put the rigid displacements in a form compatible with the acoustic code. (Working with rotational motion, however, as with parachute pendulum modes, is not so easy to describe.)

One then solves the eigenvalue problem describing coupled oscillations of the structure and fluid field, and infers the mass of the fluid. The coupled equations developed for the structure and the fluid are

$$
\left[\begin{array}{cc}
K_{S}-\omega^{2} M_{S} & C^{T} \\
C & \frac{H(k)}{\rho_{l} \omega^{2}}
\end{array}\right]\left\{\begin{array}{l}
\boldsymbol{U} \\
\boldsymbol{Q}
\end{array}\right\}=\left\{\begin{array}{l}
\boldsymbol{F}_{S} \\
\boldsymbol{F}_{A}
\end{array}\right\}
$$

where $\omega$ is the frequency of oscillation of the structure and fluid acting together. $F_{S}$ is a structural force vector and $F_{A}$ is the acoustic excitation (incident acoustic field). ${ }^{+}$

The added mass matrix is found by elimination of $Q$ from Eq. (5) and assuming free vibration (zero force on right-hand side):

$$
\{\boldsymbol{Q}\}=-\rho_{r} \cdot \omega^{2}[H(k)]^{-1}[C]\{\boldsymbol{U}\}
$$

This is substituted into the upper equation in Eq. (5) to obtain:

$$
\left(\left[K_{S}\right]-\omega^{2}\left(\left[M_{S}\right]+\left[M_{A}(k)\right]\right)\right)\{\boldsymbol{U}\}=\{\mathbf{0}\}
$$

where

$$
\left[M_{A}(k)\right]=\rho_{l}[C]^{T}[H(k)]^{1}[C]
$$

is the added mass matrix. To this point, the added mass is frequency dependent, so that the eigenvalue problem in Eq. (7) is not a conventional algebraic eigenvalue problem. We now, however, assume that the fluid is incompressible, i.e., $k$ is zero. This ensures synchronous motion of the entire fluidsolid system. The added mass can be shown to be real in this case. (If $k \neq 0$, the motion will not be synchronous, and will have traveling-wave character.) Equation (7) can then be solved using subspace iteration, for example.

The added mass matrix $\left[M_{\lambda}\right]$ is a full symmetric matrix due to the use of the boundary element model for the fluid. The banded character of the original structural mass matrix $\left[M_{S}\right]$ is therefore of little value, because bandedness is lost in the assembled system matrix.

There has been conflicting use of the words "added mass" and "virtual mass" in the literature. Hydrodynamicists" have used added mass to describe the fluid contribution only, and virtual mass to refer to the total mass of the structure and fluid. Aeroelasticians, "however, have used virtual mass to apply to the fluid contribution only. In this article the term virtual mass will be avoided because it has been compromised by the two usages. The term added mass will be used for the fluid contribution, alone.

\section{Preliminary Studies (Classical)}

To develop some feeling for the added mass calculations, one can repeat some classical cases. This will also aid in determining the mesh refinement needed.

The first case studied is a thin, rigid, circular disk of $1 \mathrm{~m}$ radius, moving normal to its plane in air at standard sea level conditions (assumed inviscid and incompressible). This case is difficult numerically because of the sharp edge of the disk. The fluid velocity around this sharp edge reaches infinity. This "potential" singularity is similar to the leading-edge singularity in subsonic thin-airfoil theory. Care must be taken to set the pressure jump across this cut edge to zero. The solution for the added mass of a thin disk is given by Lamb5:

$$
M_{A}=2.6667 \mathrm{~kg}
$$

Because of the potential singularity at the cut edge, it is important to have a layer of narrow elements close to that edge (Figs. 1-3). Three mesh densities were used to study convergence. Because the outer nodes are placed on the reference diameter, the disk area is slightly underestimated.

Added mass results for the disk are given in Fig. 4. The error consists of two major parts: 1) that due to underestimation of the disk area and 2) that due to the interpolation error inherent in a coarse mesh. The fine mesh has less than $1 \%$ error in added mass.

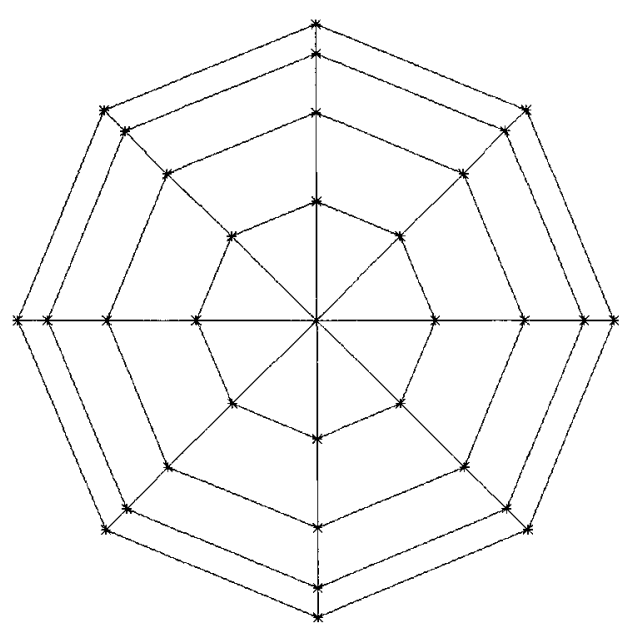

Fig. 1 Coarse mesh for circular disk.

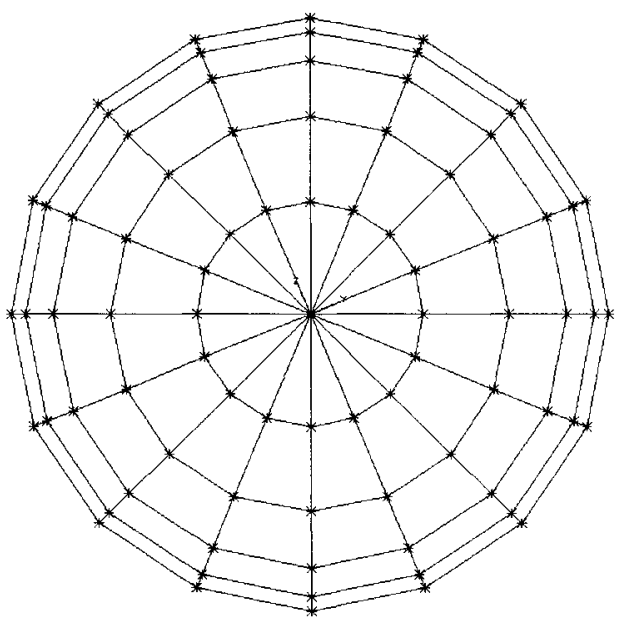

Fig. 2 Medium mesh for circular disk. 


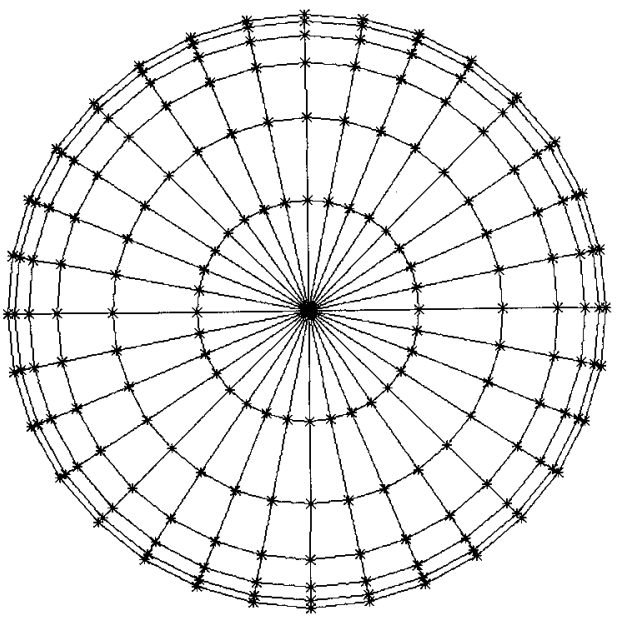

Fig. 3 Fine mesh for circular disk.

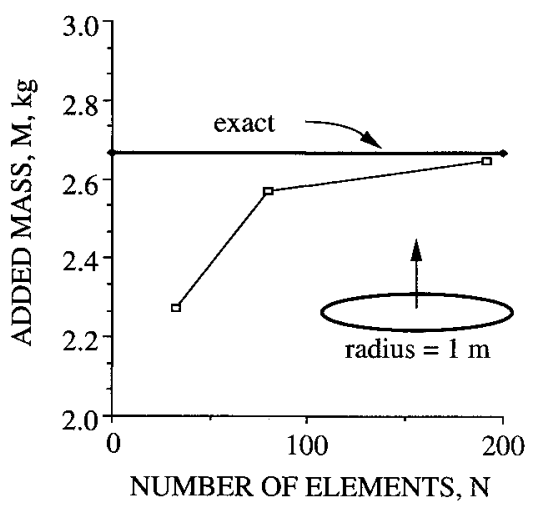

Fig. 4 Mesh refinement study for circular disk.

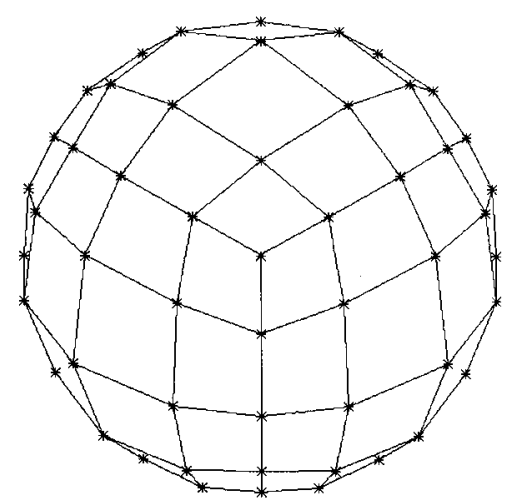

Fig. 5 Coarse mesh for sphere, with hidden lines removed.

The second case studied is a sphere. The general problem can be solved in closed form. ${ }^{5}$ The added mass is found to be one-half the displaced fluid mass. This holds for accelerations in all directions, of course. The sphere has no "cut edges," and there is no need to apply side conditions on pressure jump. A coarse mesh is shown in Fig. 5.

The nodal points for the boundary elements are placed on the reference diameter. The boundary elements form a faceted surface that lies inside the reference sphere, and hence, displaces a smaller amount of fluid than the reference sphere. As a result, the "raw" answer obtained for added mass is for a smaller body than the reference sphere. An inertia coefficient is defined for a perfect sphere as the ratio of added mass to enclosed mass (of the same fluid). One can correct the inertia coefficient by using the actual enclosed mass in the faceted sphere as the reference, rather than the mass of a perfect sphere. This reduces the convergence issue to one

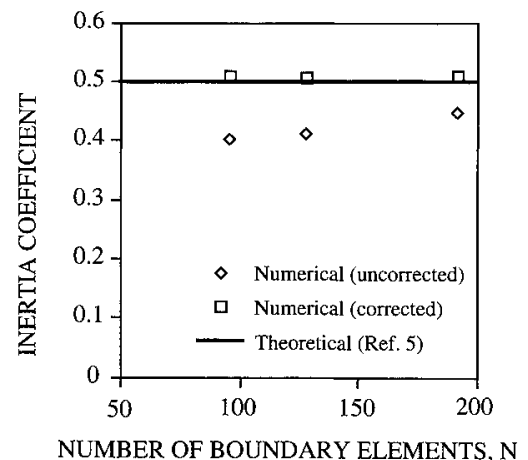

Fig. 6 Inertia coefficient for sphere, with and without volume correction.

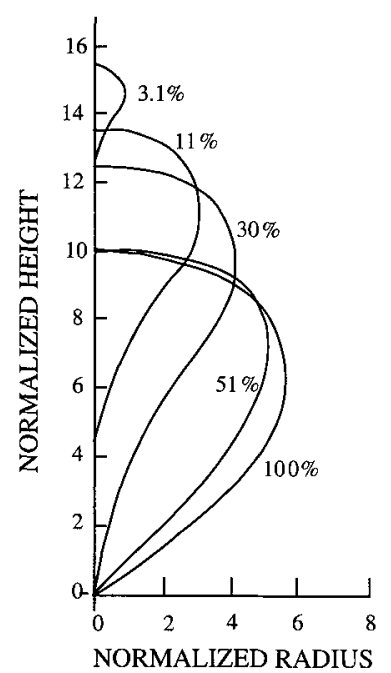

Fig. 7 Cross sections of balloons for varying inflation. ${ }^{7}$

involving the coarseness of the mesh, and removes the question of the reference volume. Convergence studies (below) for the balloon also show better convergence with such volume correction.

The use of volume-correction is also needed for a second reason. The boundary element solution for the sphere includes not only the outer fluid field, but also the (same) fluid within the sphere. This internal air mass is not appropriate in our case since actual balloons carry helium, not air, and must be subtracted out. The mass of helium must then be added in. The inertia coefficient calculation is sensitive to volume error if this correction is not done.

An acoustic study was done for the sphere using SYSNOISE with three boundary element mesh densities (Fig. 6). Volumecorrected inertia factors for all meshes were within $1 \%$ of the analytical value of 0.5 . This shows the effectiveness of the volume correction, because the displaced volume of the coarsest mesh was $8 \%$ lower than the smooth reference sphere.

\section{Balloon Studies}

A family of balloons of the Smalley type (zero circumferential stress) will be considered. Five balloon inflation ratios will be considered. Rand ${ }^{7}$ has provided a procedure to size the balloons for given inflation ratios. This procedure will be used to lay out the balloon shapes (Fig. 7) corresponding to inflation fractions from 0.0031 to 1.000 .

The balloon mesh was constructed by creating a $\frac{1}{8}$ section of the balloon and then replicating it eight times with the preprocessor I-DEAS. The completed model is faceted (e.g., with flat elements), i.e., no attempt was made to account for the scalloped nature of the segments as the film bulges from the load tapes. 
The "float" (fully inflated) balloon case has been studied intensively, in order to resolve the mesh density issue. Figures $8-10$ show the three mesh densities. From the experience with the sphere, one would expect this more complicated body to be adequately modeled with 256 elements (medium mesh), and rather accurately modeled with 432 elements (fine mesh).

The float results for added mass in the vertical (on-axis) and horizontal (lateral) directions are given in Table 1. The difference between the fine and the medium mesh is 2.8 and

Table 1 Inertia coefficients for balloons at float

\begin{tabular}{llll}
\hline Mesh & Coarse & Medium & Fine \\
\hline Elements & 112 & 256 & 432 \\
Nodes & 114 & 226 & 218 \\
Enclosed volume & 1.6416 & 1.7152 & 1.7262 \\
Raw vertical inertia & 0.540 & 0.599 & 0.592 \\
Raw horizontal inertia & 0.337 & 0.380 & 0.387 \\
Corrected vertical incrtia & 0.673 & 0.663 & 0.645 \\
Corrected horizontal inertia & 0.453 & 0.435 & 0.433 \\
\hline
\end{tabular}

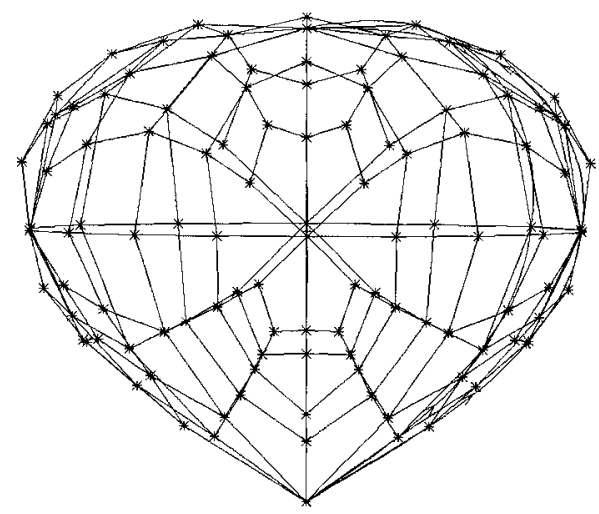

Fig. 8 Coarse mesh for balloon at float.

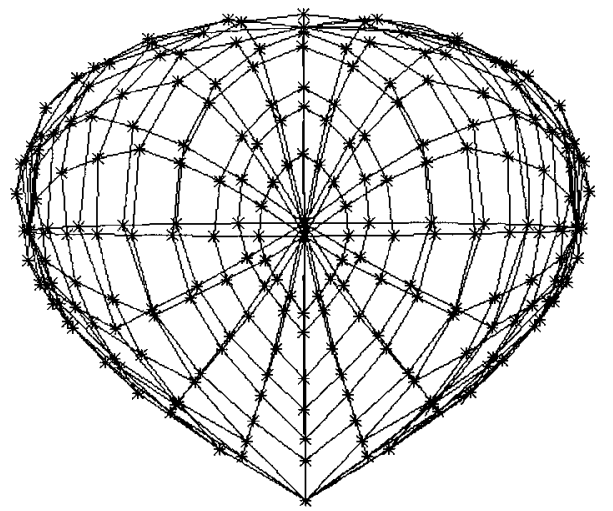

Fig. 9 Medium mesh for balloon at float.

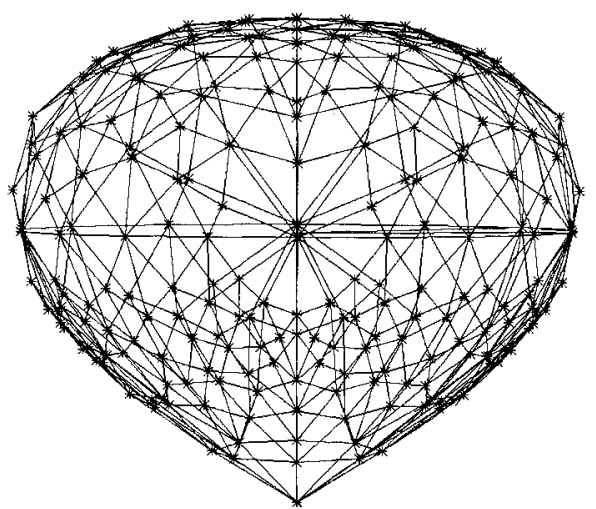

Fig. 10 Fine mesh for balloon at float.
$0.5 \%$ for the axial and lateral cases, respectively. Perhaps the results are converging more slowly on axis because the balloon presents a "flatter" (more bluff) profile in that direction.

Convergence is from above, therefore, the true values for added mass will be slightly lower than the fine mesh result. For engineering purposes, the medium mesh is sufficient to study the partially inflated family of balloons.

Results for a family of partially inflated balloons are plotted in Fig. 11. The full balloon has one characteristic of the flat disk, i.e., the vertical (on-axis) inertia is higher than the horizontal (lateral). The inertial coefficients range from 0.42 to 0.64 , depending on the inflation and the orientation of acceleration.

It is useful to have an analytical expression for the added mass, for use in balloon trajectory computer programs. The best fit for vertical added mass is a cubic, as shown in Fig. 12:

$$
y(x)=0.39967+0.47881 x-0.30341 x^{2}+0.069664 x^{3}
$$

The coefficient of determination $r^{2}$ is a measure of quality of fit, where 1.0 represents an ideal fit."

The best fit for horizontal added mass is a quadratic, shown in Fig. 13:

$$
y(x)=0.64944-0.50739 x+0.29101 x^{2}
$$

The erratic nature of the coefficients at small inflation frac-

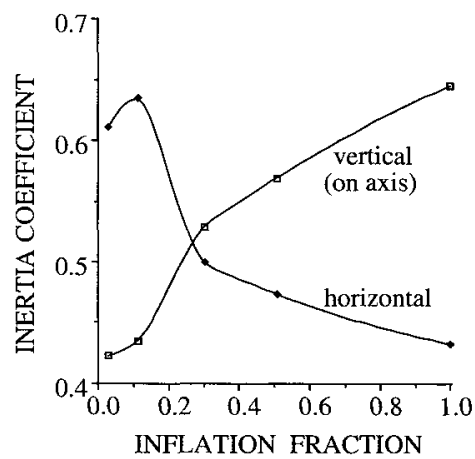

Fig. 11 Added mass for high-altitude balloon.

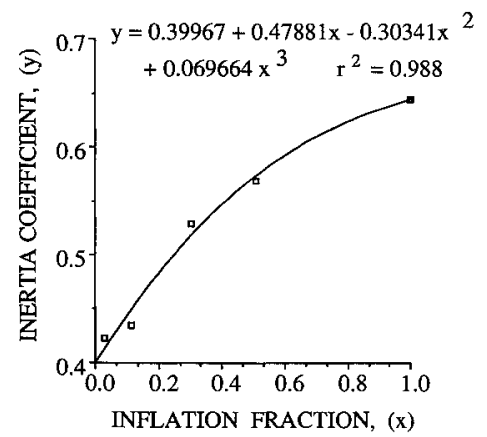

Fig. 12 Cubic fit to vertical added mass for balloon.

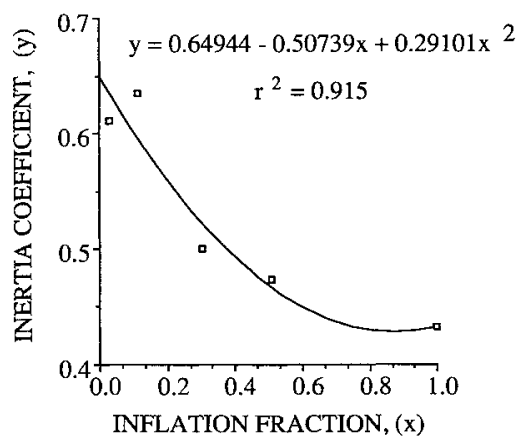

Fig. 13 Quadratic fit to horizontal added mass for balloon. 
tions is due to the difficulty in defining the small bubble at the end of a long "rope" of collapsed polyethylene film. The volume of the rope has been neglected in these calculations. The values for added mass at small inflation fractions should be viewed as having less precision.

To date there have been limited experimental results to compare with this theory. The NASA/GSFC Wallops Flight Facility has flown tethered small-scale balloons and has measured inertia coefficients for vertical acceleration of approximately $0.55 .^{8}$ In the past, researchers often applied the value of 0.5 for spheres to the study of high-altitude balloons. ${ }^{10}$ The current values will allow added mass to be adjusted continuously with flight conditions (fraction of inflation). Although there is little experimental confirmation of the acoustic theory for added mass, the infinitesimal acoustic theory is on a similar firm foundation as the infinitesimal theory of elasticity, so no surprises are expected.

\section{Computational Details}

Three software packages were used for this study. The IDEAS preprocessor was used to generate the mesh. MSCl NASTRAN was used to generate the rigid body mode information. SYSNOISE was used for the numerical acoustics. An alternate approach would have been to use ANSYS for the generation of the mesh and modal information and SYSNOISE for the acoustic analysis.

The computers used were Apollo 3500 workstations, on the University of Michigan Computer Aided Engineering Network (CAEN). The computer time expended was far greater for mesh generation and modal analysis than for the acoustic solution.

\section{Concluding Remarks}

A numerical method has been used to find added mass for high-altitude balloons. The method will be useful for other bodies immersed in fluids, whether planar or solid bodies. One must account for the presence of sharp edges (and the need for a zero pressure jump across the edge) and the presence of fictitious interior fluid in solid bodies.

The specific inertia coefficients obtained for high-altitude balloons will help in developing flight simulation codes that model dynamic balloon behavior. Common sources of excitation include ballast drops that cause axial acceleration and side winds that cause lateral accelerations. The inertia coefficients obtained range from 0.43 to 0.64 , depending on inflation and direction of acceleration. These new values should replace the classical value of 0.5 for a sphere, which has been used in many calculations to date.

\section{Acknowledgments}

The computer software used in this article was made available through University programs from Numerical Integration Technologies (SYSNOISE), Structural Dynamics Research Corp. (I-DEAS), and the MacNeal-Schwendler Corp. (MSC/ NASTRAN). The use of these programs is greatly appreciated. Technical comments have been received by Jean-Pierre Coyette and Peter VanVooren of Numerical Integration Technologies.

\section{References}

'Zienkicwicz, O. C., Kelly, D. W., and Bettess, P., "The Coupling of the Finite Element Method and Boundary Solution Procedures," International Journal for Numerical Methods in Engineering, Vol. 11, No. 2, 1977. pp. 355-375

Huang. H., "Helmholtz Integral Equations for Fluid-Structure Interaction," Advances in Fluid-Structure Interaction, Vol. 64, American Society of Mechanical Engineers, Applied Mechanics Div., New York. 1984, pp. 19-38.

Ousset, Y., and Sayhi, M. N., "Added Mass Computations by Integral Equation Methods," International Journal for Numerical Methods in Engineering. Vol. 19, No. 9, 1983, pp. 1355-1373.

+SYSNOISE Theoretical Mamual, Version 4.3, Numerical Integration Technology, Heverlee, Belgium, July, 1990, pp. 3-8.

Lamb, H., Hydrodynamics, 6th cd. Dover, New York, 1945, pp. 123. 124 .

"Bisplinghoff, R. L., Ashley, H., and Halfman, R. L., "Aeroclasticity," Addison-Wesley, Reading, MA, 1955, p. 200.

'Rand, J.. "Shape and Stress of an Ascending Balloon," Winzen International, Inc., Rept. WII-9936-FR-01, San Antonio, TX, June 1987.

"Robbins, E., and Martone, M., "Determination of Balloon Gas Mass and Revised Estimates of Drag and Virtual Mass Coefficients," Committce on Space Research XXVII Plenary Meeting and Associated Activities, The Hague. The Netherlands, July 1990.

'CA Cricket Graph III User Guide, Computer Associates International. Islandia. NY, 1992, p. 202.

"Anderson, W. J., and Taback, I., "Oscillations of High-Altitude Balloons," Journal of Aircraft, Vol. 28, No. 9, 1991, pp. 606-608. 